\title{
Anaesthetic requirements in patient monitoring
}

\author{
J. P. PAYne \\ Research Department of Anaesthetics, Royal College of Surgeons of England, \\ Lincoln's Inn Fields, London, W.C.2
}

\begin{abstract}
Summary
The differences between monitoring the unconscious patient in the operating theatre and the semi-conscious patient in the recovery room are stressed.

In the semi-conscious patient particular difficulties arise at the patient-sensor interface and with datahandling procedures which often disturb the patient.

New methods of data processing, including the use of computers, are discussed.
\end{abstract}

\section{Introduction}

According to the New English Dictionary, to monitor means to give warning of impending danger. Fundamentally monitoring techniques in anaesthesia serve three main purposes. First they protect the patient by ensuring that the anaesthetist is made aware of any disturbance in physiological patterns beyond acceptable limits. Secondly, the pattern of the patient's response to the effects of anaesthetic and allied drugs or to the effects of surgical interference can be assessed with a view to reversal, curtailment or withdrawal if necessary. Thirdly, the data derived from monitoring specific groups of patients can be used to provide a better understanding of the effects of a given technique or procedure.

In anaesthesia the problems of monitoring are conveniently considered under two headings, those related to the operating room and those concerned with management in the post-operative phase in the recovery ward or the intensive care unit.

\section{Monitoring in the operating theatre}

Monitoring problems in the operating theatre are of no great complexity since the patient is usually unconscious and motionless and the main limitation is the availability or otherwise of suitable equipment. Indeed the operating room provides a better opportunity for comprehensive monitoring than any other clinical environment.

Traditionally the anaesthetist is interested in circulatory and respiratory patterns and until recently was content to measure the heart rate, the blood pressure and the respiratory rate. Today he has a wide choice of physiological and biochemical parameters which not only provide information about the circulation and respiration but also deal with awareness, body temperature, muscle tone, acid-base and electrolyte balance and renal function.

Electrocardiography. Perhaps the most popular circulation monitor in the operating room is the electrocardiograph, the use of which was pioneered by Johnstone (1948). In addition to providing information about the rate and rhythm of the heart the electrocardiogram will also indicate the presence or absence of effective coronary blood flow, disturbances in calcium and potassium ion concentrations and occasionally drug intoxication. For most monitoring purposes during anaesthesia a single channel oscilloscope with a $12 \mathrm{~cm}$ screen is suitable but some form of print-out is useful if a permanent record is needed. Most anaesthetists prefer to use lead two of the standard leads and because electrical interference is common in the operating theatre particular care is needed in the selection of electrodes and in the proper connection to earth.

A further difficulty is the problem of trailing leads which tend to become entwined in other equipment and to provide hazards for the theatre staff. In either case they become disconnected, if not broken. The most satisfactory method of dealing with this problem is to convert to radio-telemetry (Holter, 1957). Small transistorized transmitters no larger than a matchbox have been designed with a range of up to $50 \mathrm{ft}$ and are ideally suited for operating theatre and recovery room monitoring.

Blood pressure measurements. Many indirect techniques are available for the measurement of blood pressure but they all suffer from the disadvantage that they are least effective when most needed. The difficulty of measuring blood pressure in patients suffering from haemorrhagic shock or after severe trauma is well known; direct measurement with a catheter in a suitable artery is the only certain way to measure blood pressure accurately. This raises problems of technique, risk, equipment, cost and not least ethics. 
Modern arterial catheterization techniques based on Holmgren's modification (1956) of Seldinger's method (1953) allow percutaneous insertion into vessels such as the radial artery with minimal disturbance to the patient. Open mobilization of the artery is rarely needed and certainly there seems little justification for ligation even should exposure become necessary. Moreover the non-irritant quality of modern plastic materials ensures that little trauma results from catheters maintained in position over a prolonged period. Equipment for the direct monitoring of blood pressure can be both bulky and expensive but for routine purposes a simple inexpensive aneroid manometer such as that described by Mallard, Payne \& Peachey (1963) has proved satisfactory. Systolic, diastolic and pulse-pressures are easily followed and disturbances of ventricular rhythm are equally obvious. A further advantage of direct arterial monitoring is that by provision of a three-way tap between the catheter and the transducer or manometer serial samples of arterial blood can be taken for respiratory, acid-base and electrolyte assessment as well as for the determination of anaesthetic concentrations in the blood.

Consideration of these facts puts the ethical problem in perspective. The objections raised by some doctors to arterial catheterization must be difficult to sustain when the advantages are so obvious for those critically ill patients for whom monitoring is virtually mandatory. If it could be shown that arterial catheterization in itself carried a definite risk however small then such objections might be justified but in more than 20 years experience of intra-arterial blood pressure measurements the author has yet to experience a major mishap attributable to the technique.

Blood flow measurements. There is however scope for questioning the attention given to blood pressure measurements during anaesthesia. The fact that the blood pressure is often deliberately lowered during surgery and that levels of $50 \mathrm{mmHg}$ and lower may be maintained for many hours suggests that at least in the conditions existing in the operating theatre other circulatory criteria may be of more significance. In particular, tissue perfusion measurements would seem to offer a valuable guide to the patient's circulatory state. Unfortunately such measurements are not easy under clinical conditions; for the most part they are limited to semi-quantitative determinations of finger blood-flow using photoelectric cells or carbon microphones; more sophisticated air-displacement and water-displacement plethysmographs have been developed to measure finger, hand and forearm blood-flow but until the problems of bulk and calibration have been solved they are likely to remain experimental tools.

Central venous pressure. In patients suffering from severe blood loss or whose fluid balance is otherwise disturbed the central venous pressure is probably the most useful guide not only to replacement therapy but also to the ability of the right heart to handle the venous return (Sykes, 1963; Hardaway, 1968). Venous pressure can be measured by any hollow tube inserted into any part of the venous system provided that, first, there is unobstructed flow between the point of entry and the heart and, second, the reference point remains constant. For monitoring purposes the fast fluctuations in venous pressure induced by the contraction of the heart muscle and by the respiratory process are less significant than the slow change in static pressure attributable to the venous return. Thus once again expensive equipment is only warranted if permanent records are required.

Blood volume and cardiac output. The presence of arterial and venous catheters makes the measurement of circulating blood volume and cardiac output easy and repeated determinations can be made at no risk to the patient. With modern dye-dilution techniques the extraction of dye is no longer necessary and the cardiac output result can be available within 2 min of the injection of the dye.

Blood analysis. But access to arterial and central venous blood has other advantages in monitoring. As already indicated the assessment of respiratory and acid-base disturbances is simplified. In particular, determinations of oxygen and carbon dioxide tension provide a more accurate evaluation of respiratory efficiency than measurements of tidal and minute volume even though there may be a few minutes delay while the analyses are being carried out. Respiratory changes can be monitored directly during anaesthesia by means of a Wright anemometer or if permanent records are required by means of a Fleisch pneumotachograph. More simply a spirometer with a recording drum attachment can be substituted for the reservoir bag on the anaesthetic machine. If a closed system is being used it is also possible to measure the oxygen consumption without modifying the circuit.

Electroencephalography. The question of awareness in anaesthesia is a recurring problem especially when light anaesthesia is employed combined with neuromuscular blocking agents. At first sight the electroencephalograph was the obvious solution to this problem but in practice certain difficulties arose. First, for detailed information several channels are needed, second the interpretation of the electroencephalogram is essentially a matter for the expert; third, patterns are influenced by other factors such as hypotension and hypothermia, and, fourth, artefacts and other interference may distort the pattern and make interpretation difficult. However, recent experiments with computer analysis suggest that a programme can be devised to remove artefacts and 
other interference which should enable the anaesthetist to follow the pattern associated with different depths of anaesthesia, hypotension and hypothermia on a single channel.

Temperature measurements. The development of hypothermic techniques for cardiac, vascular and neurosurgery stimulated a renewed interest in temperature changes in the body and suitable transistorized probes have been designed to measure the temperature in the nasopharynx, the oesophagus and rectum, as well as on the skin surface and in muscle. All these temperatures can be measured simultaneously and continuously but most commonly each probe is scanned in turn and the value charted either automatically or manually from a common meter.

\section{Monitoring in the recovery room}

The availability of so many and varied techniques would seem to imply that monitoring in the recovery ward and in the intensive care unit was merely a matter of selecting the appropriate technique. In practice the problems that arise in monitoring critically ill patients who may be restless, semiconscious and unco-operative are substantially greater than those associated with the unconscious, motionless patient in the operating theatre. The difficulties are associated partly with the patientsensor interface because most monitoring equipment involves some limitation or interference at a time when the patient is least willing or able to tolerate it and partly with the data-handling procedures which only too often obtrude on the patient's awareness.

The monitoring of respiration is an excellent example; although the quantitative assessment of respiratory function is easy in the physiological laboratory and in the operating theatre the equipment is complicated and usually unsuited for use in intensive care situations. The simplest test of respiratory function is to determine the rate of breathing and while this can obviously be done by inspection it is not very practical if breathing is to be monitored continuously. For this purpose a thermistor probe placed at the nostril can be used without disturbing the patient unduly; alternatively an air-displacement pneumograph fixed around the chest-wall is also effective. For a more sophisticated assessment of ventilation the use of an electrical impedance pneumograph has been proposed (Geddes et al., 1962). This instrument measures the changes in transthoracic impedance induced by the various phases of respiration. The impedance as indicated by the voltage is linearly related to the volume of air in the lungs and provided that separate calibration is carried out for each patient the respiratory pattern will be recorded accurately. The electrical impedance pneumograph has the advantage that it is indepen- dent of face-masks and tracheal tubes; moreover, the same electrodes can be used to derive the electrocardiogram which although an unconventional lead clearly indicates disturbances in heart rate and rhythm (Baker \& Hill, 1969).

In an attempt to overcome some of the difficulties involved in long term monitoring special harnesses have been designed to carry the various leads needed to measure such parameters as heart rate, ECG, respiratory patterns and temperature at different sites. None has proved entirely satisfactory and when taken in conjunction with the fact that cardiovascular and respiratory parameters incorporate some form of alarm system to give warning when the values fall outside certain preset readings it is not surprising that the percentage of false alarms is high. Even when delay mechanisms are introduced and when the alarm is programmed to respond only after more than one parameter has gone beyond normal limits, false alarms are common and do much to destroy the confidence of the nursing staff. In this connection it is imperative that warning systems are seen to work efficiently otherwise the nurse gains the impression that not only has she the care of critically ill patients but she also has the responsibility of looking after some not very dependable machinery. Furthermore her sense of vocation is not strengthened by the suggestion in some circles that the proper use of monitoring equipment will reduce the number of nurses required in recovery wards and intensive care units. It cannot be stressed too emphatically that the purpose of monitoring is not to replace the nurse but to make her task easier by relieving her of the almost perpetual anxiety imposed by the need for constant alertness.

\section{Data processing}

Data-handling procedures vary slightly according to whether a central monitoring system is installed or whether reliance is placed on individual bedside units, but with either system the basic problem is that of presenting information to the doctor or the nurse without disturbing the patient. The cathode ray oscilloscope provides a satisfactory visual display unit but of itself does not draw attention to abnormalities. Similarly print-out data sheets have to be scanned to detect any deterioration. Audio signals help the nurse but especially with bedside units may also be heard by the patient, a few of whom develop a 'beat dependence' in that they cannot bear to be separated from the sound of their own heart beat. Probably the combination of an oscilloscope with a warning light is the best compromise.

Data analysis poses further problems. In the past the scope of many clinical investigations was limited by inability to process the relevant data sufficiently 
rapidly and accurately. Such difficulties are gradually being overcome by present technological advances; in particular the advent of the digital computer has provided new opportunities for data processing. Before the computer can handle data, however, the material must reach it; unfortunately few operating rooms and intensive care units have a computer immediately available. In an attempt to overcome this problem the possibility of using a direct telephone link from the operating room to the computer laboratory was investigated. It was established that physiological signals such as the ECG, the blood pressure and the respiratory pattern could be transmitted in analogue form over substantial distances (Colbeck et al., 1968). The next step was to determine whether or not such signals could be sent simultaneously and in September 1968 during the Fourth World Congress of Anaesthesia the ECG and blood pressure contours of a patient in the recovery ward of the University Hospital in Nijmegen, Holland, were transmitted simultaneously over the switched international telephone network to the Festival Hall, London (Hill et al., 1970). Since then a three-channel link has been established between St Peter's Hospital in Henrietta Street, London, and the computer laboratory in the Research Department of Anaesthetics in the Royal College of Surgeons (during the course of this lecture the ECG, blood pressure and respiratory patterns of a patient in the recovery ward in St Peter's Hospital were transmitted simultaneously to the lecture room). The details of the system will shortly be published.

The success of these transmissions has brought the use of computers for on-line and rapid turn-around analysis during anaesthesia and in intensive care situations a step nearer. Current work is concerned with preparing the relevant programmes for handling such data. Already an arrhythmia programme is available and a simple blood-pressure programme is nearing completion.

It is obvious that despite the developments in technology and the variety and range of equipment continuous monitoring of severely ill patients raises many problems. But the basic difficulty is the need to marry two almost incompatible requirements; that of freedom of movement for the patient and that of intimate contact of instruments with blood and tissue. The position is not improved by the tendency of clinicians and designers alike to concentrate on parameters that are easily measured rather than on the more difficult measurements which may have more to offer. The future pattern of patient monitoring will be decided by the ability or otherwise of clinicians and designers to meet these requirements and this in turn will require more co-operation than has hitherto occurred.

\section{References}

BAKER, L.E. \& HiLl, D.W. (1969) The use of electrical impedance techniques for the monitoring of respiratory pattern during anaesthesia. British Journal of Anaesthesia. 41, 2.

Colbeck, W.J., Hill, D.W., Mable, S.E.R. \& Payne, J.P. (1968) Electrocardiographic transmissions by public telephone. Lancet, ii, 1017.

Geddes, L.A., Hoff, H.E., Hickman, D.M. \& Moore, A.G. (1962) The impedance pneumograph. Aerospace Medicine, 33, 28.

HaRdaway, R.M. (1968) Clinical Management of Shock. Charles Thomas, Springfield, Illinois.

Hill, D.W., Payne, J.P. \& Crul, J.F. (1970) Patient monitoring by telemetry. In: Progress in Anaesthesiology. p. 361. Excerpta Medica International Congress Series No. 200.

Holmgren, A. (1956) Circulatory changes during muscular work in man. Scandinavian Journal of Clinical Laboratory Investigation, 8, Suppl. 24.

HOLTER, N.J. (1957) Radioelectrocardiography: a new technique for cardiovascular studies. Annals of the New York Academy of Sciences, 65, 913.

JoHNSTONE, M. (1948) The effects of general anaesthetic agents on abnormal hearts-a study in electrocardiography. Thesis, Queen's University, Belfast.

Mallard, J.R., Payne, J.P. \& Peachey, C.J. (1963) Continuous monitoring of blood pressure by intra-arterial catheterization of the radial artery using a watch-type aneroid manometer. Journal of Physiology, 167, 10-11P.

SELDINGER, S.I. (1953) Catheter replacement of the needle in percutaneous arteriography. Acta Radiologica (Stockholm), 39, 368.

SYKES, M.K. (1963) Venous pressure as a clinical indication of adequacy of transfusion. Annals of the Royal College of Surgeons of England, 33, 185. 